Collaborative Research Program: general effectiveness of treatments. Arch Gen Psychiatry 1989; 46(11): 971-82.

8 Morrison PD, Murray RM. The antipsychotic landscape: dopamine and beyond. Ther Adv Psychopharmacol 2018; 8(4): 127-35.

doi:10.1192/bjb.2018.32

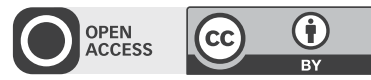

(c) The Authors 2018. This is an Open Access article, distributed under the terms of the Creative Commons Attribution licence (http://creativecommons.org/ licenses/by/4.0/), which permits unrestricted re-use, distribution, and reproduction in any medium, provided the original work is properly cited.

\section{Rational antidepressant use}

In her contribution to the 'Against the Stream' series,

Dr Moncrieff ${ }^{1}$ articulates the case for the drug-centred model of antidepressant action. She notes that antidepressants do not typically outperform placebo in well-designed studies (particularly in rare instances where an active placebo is used as a control $^{2}$ ), have little clinical effect and can cause serious adverse effects. Having made the case that antidepressants are not 'specific' antidepressant agents, she makes some comments about their use in clinical practice. I would like to offer a few remarks about these issues, including some musings about what 'rational antidepressant use' might look like.

Modern psychiatric practice has seen the rise and fall of several promising antidepressant agents (the monoamine oxidase inhibitors, the tricyclic antidepressants and selective serotonin reuptake inhibitors (SSRIs)). Recent efforts include testing the possible antidepressant properties of ketamine. But are these efforts futile? Perhaps yes, perhaps no. A truly specific antidepressant drug (if one is ontologically possible) appears to be a pipedream, given current diagnostic limitations. Our categorisation of major depressive disorder is highly heterogeneous, ${ }^{3}$ creating a disjunctive category of cognitive, behavioural and biological symptoms that do not reliably cluster together. Even if any of our current drugs had specificity for 'depression', this would be extremely difficult to uncover in clinical practice or research settings. As a result, drug development will be prone to ideological, as opposed to scientific, revolutions. ${ }^{4}$

Should we therefore abandon antidepressants as a treatment modality? As long as we are honest with our patients about our current state of knowledge, I think not. Drug use has always been an integral part of human life, ${ }^{5}$ helping to alleviate life's various physical, emotional and existential pains. Antidepressants are no different in this respect. While researchers continue the search for a discrete condition called 'depression', drugs such as the SSRIs can be exploited for particular patient complaints. Antidepressants can cause emotional blunting, sedation, activation and decreased libido, among other things. Some have a proclivity towards one effect more than others. These effects can be exploited to relieve particular problems (e.g. sedation to alleviate insomnia, or emotional numbing to transcend an episode of intense anxiety or distress), without pretence towards a yet-to-be discovered condition. A rational provider would match a drug's effects to the patient's complaints, irrespective of diagnosis (or drug class); and would remain vigilant to the development of any adverse effects or deterioration of condition, start at the lowest recommended dose, and withdraw the patient from the drug as soon as possible. Psychosocial interventions can remain an important part of treatment, in many cases being the first treatment of choice. Antidepressants, like all drugs, are neither angels nor demons. They should be used selectively and thoughtfully, when used at all.

Daniel Dunleavy, MSW, Doctoral Candidate, Florida State University College of Social Work; email: djd09e@fsu.edu

1. Moncrieff J. Antidepressants are not antidepressants - an alternative approach to drug action and implications for the use of antidepressants. BJPsych Bull 2018; 42(1): 42-4.

2. Jensen JS, Bielefeldt AS, Hróbjartsson A. Active placebo control groups of pharmacological intervention were rarely used but merited serious consideration: a methodological overview. J Clin Epidemiol 2017; 87: 35-46.

3. Paris J. The mistreatment of major depression. Can J Psychiatry 2014; 59 (3): 148-51.

4. Healy DT. The structure of psychopharmacological revolutions. Psychiatr Dev 1987; 4: 349-76.

5. Chast F. A history of drug discovery. In The Practice of Medicinal Chemistry (ed CG Wermuth), 3rd edn. Academic Press, 2008.

doi:10.1192/bjb.2018.33

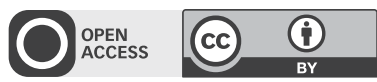

(c) The Author 2018. This is an Open Access article, distributed under the terms of the Creative Commons Attribution licence (http://creativecommons.org/ licenses/by/4.0/), which permits unrestricted re-use, distribution, and reproduction in any medium, provided the original work is properly cited.

Author's reply: In response to Dr Jauhar and Professor Young, I am used to being accused of using ideology, of being selective, of not being balanced or of being polemical. I take no personal offence, but it is important to point out that this is a useful tactic if you want to shut down debate. It harnesses the authority of science to present one view as neutral, objective and credible, and the other as self-interested and unreliable. In truth, we all bring assumptions and biases to our work. I am obviously unable to describe every study ever done on antidepressants in a short article, but I have written books and papers that address all the evidence I could find that supports the disease-centred model of drug action in relation to antidepressants and other psychiatric drugs. ${ }^{1}$

Indeed, one of the most important points I am making in relation to drug action is that existing psychopharmacological research is based on unexamined assumptions about how drugs work. These consist of the idea that drugs target the neurological mechanisms underlying symptoms, whether the latest theory about mechanisms concerns abnormalities of neurotransmitters, neural networks or neuro-plasticity. This idea has allowed psychopharmacology research to ignore the alterations to normal functioning that psychiatric drugs produce, and that will affect mental states including mental disorders, regardless of the underlying mechanisms.

Jauhar and Young point out that the latest meta-analysis of antidepressant trials finds impressive odds ratios for effects of antidepressants, but it analyses categorical outcomes derived 